\title{
Tangence
}

\section{Rire en poésie : parodistes, fumistes, fantaisistes}

\section{Daniel Grojnowski}

Numéro 53, décembre 1996

L’humour de la poésie

URI : https://id.erudit.org/iderudit/025924ar

DOI : https://doi.org/10.7202/025924ar

Aller au sommaire du numéro

Éditeur(s)

Tangence

ISSN

0226-9554 (imprimé)

1710-0305 (numérique)

Découvrir la revue

Citer cet article

Grojnowski, D. (1996). Rire en poésie : parodistes, fumistes, fantaisistes.

Tangence, (53), 13-27. https://doi.org/10.7202/025924ar d'utilisation que vous pouvez consulter en ligne.

https://apropos.erudit.org/fr/usagers/politique-dutilisation/ 


\section{Rire en poésie: parodistes, fumistes, fantaisistes} Daniel Grojnowski

Théoricien du langage poétique, Jean Cohen s'est naguère attaché à spécifier les relations qu'entretiennent "comique" et "poétique", deux catégories ordinairement dissociées dont il affirme qu'elles se situent de fait "en rapport d'opposition diamétrale, au double point de vue fonctionnel et structural" ${ }^{1}$. Alors que l'énoncé poétique implique le plein engagement du Sujet sur le mode pathétique qui annule toute distance dans l'expression de son rapport au monde, le comique, au contraire, rompt cet engagement et dispose une distance en refusant son implication: le comique "est négation de l'émotion, distanciation de l'objet, dé-pathétisation du monde" écrit J. Cohen avant d'articuler dans une antithèse parfaite les deux entités qu'il place sous la gouverne de l'isopatbie (pour le Poétique où le Sujet inscrit sa place dans la Nature) et de l'bétéropatbie (pour le Comique où la Nature... remet le Sujet à sa place). La paronomase poétique crée la fusion en postulant une homologie entre les signifiés et les signifiants, la paronomase comique instaure la confusion par l'explicitation d'une "contradiction axiologique interne" 2 . Au vers célèbre de Paul Éluard: "La terre est bleue comme une orange", s'oppose sa translation triviale: "La terre est bleue comme une tomate":

$$
\text { Poétique : (Sa } 1=\text { Sa } 2) \rightarrow \text { (Sé } 1=\text { Sé } 2)
$$

Comique: $($ Sa 1 = Sa 2$) \rightarrow$ (Sé $1 \neq$ Sé 2 )

En établissant des homologies entre les signifiants et les signifiés, le poétique réduit les composantes oppositives et par là même l'écart entre les termes du discours. Du même coup, il use d'un langage sacré qui s'énonce sur le mode de l'exaltation: "Le poétique est objet pathétisé parce que totalisé. ${ }^{3}$ Inversement le comique établit des dissemblances en laissant apparaître des

\footnotetext{
1 Jean Cohen, "Comique et poésie ", Poétique, n 61, février 1965, p. 49.

2 Ibid., p. 55.

3 Ibid., p. 58.
} 
éléments contraires qui se neutralisent. Il renforce l'écart entre les termes de son discours qu'il profère sur le mode de l'exultation profane. La structure du risible est invariante: "C'est toujours un processus qui se retourne contre lui-même. "4

En dépit d'une simplicité et d'une clarté toutes pédagogiques que corrobore l'efficace du binarisme, l'analyse de J. Cohen laisse perplexe à bien des égards: parce qu'elle exclut a priori de son champ des genres du poème qui traditionnellement relèvent de la vis comica (la satire, l'épigramme, la parodie); parce qu'elle se réfère essentiellement à des épisodes du cinéma burlesque (Charlot dans L'émigrant, le millionnaire des Lumières de la ville), comme si le corpus de la poésie ne pouvait fournir d'exemple comique probant; parce que le vers de Paul Éluard qui illustre à lui seul les prestiges du poétique, donne un bel exemple de fantaisie qu'éclaire un certain sourire, une fois restitué son contexte:

La terre est bleue comme une orange

Jamais une erreur les mots ne mentent pas

[...]

Elle a sa bouche d'alliance

Tous les secrets tous les sourires

Et quels vêtements d'indulgence

À la croiser toute nue

L'amour la poésie, Premièrement, IV

En opposant point par point le "poétique" au "comique ", la machine argumentative sacrifie aux impératifs de la thèse l'infinie variété des effets du poème, la diversité des tons. Elle postule qu'une essence de la poésie (une poésie de la poésie) prévaut sur ses innombrables avatars. Elle relègue à l'arrière-plan toutes les pratiques qui à différentes époques - des Grands Rhétoriqueurs aux adeptes de l'Oulipo - méconnaissent, défient ou sollicitent le Haut Langage par les exercices du jeu verbal. Elle consacre une conception de la Poésie qui la constitue comme un super-genre, procédant d'un ensemble de contraintes génériques tout en transcendant les règles de fabrique. "L'histoire des lettres est pleine de ces poésies qui sont de la prose mise en vers -

4 Ibid., p. 57. Charles Maurras avait déjà, dans un dialogue intitulé "Ironie et poésie", opposé la "poésie véritable a à toute division du ton, qui provoque la "dégénérescence": l'ironie appartient à la terre, alors que la poésie "c'est le ciel" (La Gazette de France, 12 décembre 1901, repris dans Barbarie et Poésie, Paris, 1925). 
déclare doctement un rédacteur du Dictionnaire universel de Pierre Larousse - [...] Mais la poésie n'est pas cela. Elle consiste dans un souffle qui est une émanation directe de l'âme. " 5 Apte à produire le frisson de l'indicible, le poétique procède d'un moment de grâce, il marque l'énoncé d'une empreinte particulière qui a été tour à tour dénommée: harmonie, magie, sacré, absolu. Il se concentre dans tels beaux vers dont les amateurs de "poésie pure" ont fait naguère leurs gorges chaudes, du fait que le sortilège y outrepasse la signification: "La fille de Minos et de Pasiphaé" - "Je suis le ténébreux, - le veuf, — l'inconsolé" "Orléans, Beaugency...Vendôme, Vendôme." ${ }^{6}$ Le poétique se manifeste alors non pas comme "un langage dans un langage", selon la célèbre formule de Valéry, mais au troisième degré, comme "un langage dans le langage du langage", une quintessence imprévisible et rarissime, aussi subtile qu'évanescente.

Comme la construction théorique de $\mathrm{J}$. Cohen en donne un bel exemple, nous demeurons tributaires d'une idée de la poésie qui pré-existe aux écrits, qui les discrimine et qui en affecte la lecture. Elle se perpétue dans la longue durée en organisant comme un jardin à la française un territoire aux innombrables accidents, aux parcours embrouillés. Élaborées à des fins d'enseignement, les catégorisations commandent une conception générique qui se perpétue alors même que la poétique des genres a disparu au profit de celle des époques, des écoles et des "écritures". Elle continue d'imposer un ordre et une hiérarchie, alors que l'anarchie vivante des écrits, leurs mutations, leurs continuelles hybridations rendent caduques la quête des essences.

Aristote opposait les auteurs à l'âme élevée qui s'attachent à l'imitation des belles choses, aux auteurs vulgaires intéressés par les actions des hommes vils: aux premiers sont réservés les vers héroïques, aux seconds les railleries du vers iambique! Parce qu'associé à la représentation des valeurs dégradées, le comique, tel que le conçoit sa Poétique, concerne la laideur, la difformité. Au commencement de l'ère chrétienne, le Traité du sublime renforce l'antinomie entre les fonctions triviales du discours (assimilées aux parties basses du corps, "qu'on ne peut décemment nommer") et les fonctions les plus élevées qui nous rendent

5 Article "Poésie ", t. 12, 1874, p. 1230.

6 Henri Bremond, La poésie pure, Grasset, 1926, p. 19-20 et p. 36. 
dignes du nom d'homme car la nature "a fait naître dans notre âme un amour invincible pour tout ce qui est éternellement grand et pour tout ce qui est de plus divin " 7 .

L'histoire des productions poétiques entre en discordance avec celle de l'idée de poésie qu'exposent la plupart des théoriciens. Ils associent la poésie, née d'un enthousiasme d'origine surnaturelle, au lyrisme qui la voue à remuer "l'âme des peuples par les ressorts les plus puissants " (Marmontel ${ }^{8}$ ), "La poésie est le soupir du cour qui déborde. La poésie, c'est l'univers mis en musique par le cœur", dira un poète couronné par le premier prix Nobel de littérature (Sully Prudhomme ${ }^{9}$ ). Et quand les ressources de l'effusion personnelle semblent taries, la portée métaphysique, ontologique (prière, extase, perception de la présence, communion avec le divin) prend le relais pour préserver une vocation à dire le sacré: ressortit à l'essence de la Poésie "ce qui l'assimile à Dieu même" (Paul Valéry ${ }^{10}$ ), à la prière (abbé Bremond ${ }^{11}$ ), à ce qui permet de "reprendre foi dans le monde " par la formulation de "la question de l'Etre (Yves Bonnefoy ${ }^{12}$ ). Ainsi, l'opposition qu'a établie Jean Cohen dans les termes d'une structure antinomique intemporelle, consacre une conception particulière de l'expression poétique qui procède de la tradition. Celle-ci transfère les multiples valeurs poétiques du langage en fonction messianique: inspiré par les dieux, le Poète divulgue son enthousiasme par voie de asorcellerie évocatoire". Cette conception idéaliste du poétique (idéalisé autant que spiritualisé) devenu lieu commun conforte son antagonisme avec un autre lieu commun qui réduit le comique aux procédures de dégradation et d'incongruité. L'imaginaire du "poétique "situe la suggestion, l'ineffable, le mystère, la musique, dans un au-delà des significations, aux antipodes du jeu phonique et métrique. La dissociation du signe en fonctions nobles et triviales, décalque celle du corps et de l'esprit que repro-

7 Longin, Traité du sublime, texte établi et traduit par H. Lebègue, Les BellesLettres 1952 , p. 60 et p. 50.

8 Marmontel, Éléments de littérature, article "Lyrique ", Paris, 1846, t. 2, p. 339.

9 Sully Prudhomme, Journal intime ("Pensées»), éditions Rombaldi, 1963, p. 210.

10 Paul Valéry, "Questions de poésie", CEuvres, t. 1, p. 1283, Bibliothèque de la Pléiade, 1957.

11. Henri Bremond, op. cit., p. 27.

12 Yves Bonnefoy, Entretiens sur la poésie (1972-1990), Mercure de France, 1955 , p. 21. 
duit à son tour la dichotomie de la forme et du fond (cf. le signifié linguistique, pour qui le scinde du signifiant).

Le comique du verbe poétique, chez Hugo, met en exergue les jongleries prosodiques ou le calembour, dans les Odes et Ballades, les Chansons des rues et des bois et d'autres pièces récréatives. Soucieux de faire vibrer les cordes de "toute la lyre", le génie du poète ne craint pas de déroger, sans toutefois renoncer à alterner la veine lyrique ou épique et les moments de pauses. Dans l'Avant-propos des Odes funambulesques, Théodore de Banville, bien qu'il expose clairement le projet de concilier le poétique et le comique, continue de dissocier les "ressources de notre poésie lyrique" et les jeux de la versification ${ }^{13}$. Fantaisie, bouffonnerie, parodie, relèvent d'une virtuosité apparentée, selon lui, aux excès de la caricature qui forme l'une des espèces des arts plastiques. Il revient à des commentateurs de son recueil de résoudre la contradiction: Hippolyte Babou, quand il y signale "l'alliance presque nouvelle de la fantaisie lyrique et de la fantaisie comique "14; Charles Baudelaire, quand il rappelle que la poésie contemporaine "est arrivée à un état mixte, d'une nature très complexe" qui recourt à tous les genres, tous les registres et tous les modes d'expression, au point d'invalider les distinctions traditionnelles: "le génie plastique, le sens philosophique, l'enthousiasme lyrique, l'esprit humoristique, s'y combinent et s'y mêlent suivant des dosages infiniment variés" ${ }^{15}$.

Un siècle et demi après qu'on été exposées ces pétitions de principe, on est en droit d'admettre que le conflit entre le poétique et le comique est d'ordre idéologique plus que proprement "structurel". Seuls le cautionnent des cadres hérités de l'ancienne rhétorique. L'exposé de Jean Cohen les actualise avec brio, au prix d'un arbitraire que rendent caduc les cuvres de Desnos, de Queneau, de Prévert ou de Tardieu, pour citer quelques-uns des représentants les plus remarquables de la veine poéticomique. Plutôt que de poursuivre un débat qui n'a pas raison d'être, mieux vaut observer des moments d'inquiétude où des poètes tentent de prendre en charge une contradiction qui pour n'être apparente à nos yeux fut effective aux leurs.

13 Théodore de Banville, Odes funambulesques, Paris, 1859, "Préface ", p. 2-3.

14 Ibid., p. 15 (Cette "Lettre à $M$. Théodore de Banville " a d'abord paru dans la Revue française du $1^{\mathrm{er}}$ avril 1857).

15 Charles Baudelaire, "Théodore de Banville" (Revue fantaisiste, 1861), Eutres complètes, Bibliothèque de la Pléiade, 1954, p. 1114. 
18

\section{Où gît la poésie: les expériences parodiques}

Qui veut saisir l'essence de la Poésie, doit en observer les avatars qui en perturbent sans cesse l'image. Instable comme tous les genres, elle trouve sa spécificité dans sa nature réfractaire. Depuis qu'elle s'adresse à un public limité, elle n'est pas soumise aux contraintes d'une demande strictement établie. Elle se détermine par le rejet des formules, par une remise en question qui la voue à un état de malaise endémique. Après que s'est exténué le lyrisme romantique, elle adopte le double registre de la revendication et de la dénégation, qui désigne tour à tour ce qu'elle n'est pas et ce qu'elle doit être, un mode d'expression vif-argent que toute codification dénature: "La poésie n'a d'autre but que la poésie [...] La poésie ne se définit pas." (L. Dierx ${ }^{16}$ ). Le topos de la mise à mort comme condition d'une régénérescence, appartient à un discours collectif que les proclamations juvéniles d'un Ducasse (Poésies, I et II) ou d'un Rimbaud (la lettre dite du Voyant) portent à leur paroxysme.

Pendant une dizaine d'années, de 1866 à 1876, trois publications anthologiques du Parnasse contemporain cautionnent une image respectable de la poésie qui est placée sous la gouverne de quelques auteurs de renom et d'un éditeur (A. Lemerre) qu'informe un jury qualifié. Mais sa production la plus remarquable, quoique rarement discernée, échappe à ses options : elle provient des jeux parodiques qu'elle déclenche. Parce que sceptiques, insolents ou tout simplement exclus du Cénacle, un certain nombre de jeunes poètes, proches du groupe de Nina de Villars, en dévoient l'esprit de sérieux. Leurs publications accompagnent pas à pas le Parnasse officiel en dispensant une réplique burlesque du Haut Langage qu'il voudrait consacrer: Le Parnassiculet contemporain d'A. Daudet, $P$. Arène et de quelques autres (1867, deuxième édition en 1872), l'Album zutique (1871) puis les Dixains réalistes (1876) exaltent les pratiques du poème rieuses et sacrilèges. Leur exemple sera suivi quelques années plus tard par des moqueries à l'égard de la poésie "décadente" dans Les déliquescences d'Adoré Floupette, entre autres singeries de la mode poétique ${ }^{17}$. Non pas tant qu' "à côté de toute grande ouvre, il y a une parodie" comme l'affirme Victor Hugo, mais parce que les jeunes poètes associent la quête de l'absolu aux exigences d'un questionnement. Cros, Nouveau, Verlaine ou Rimbaud, par leur engagement et leurs 
œuvres, sont des auteurs de premier ordre, mais aussi des profanateurs qui empêchent que le genre ne se fige dans un style d'école dispensateur de thèmes et de postures.

Dans leur composante la plus conventionnelle leurs parodies transposent sur le mode trivial (grossier, voire obscène dans l'Album zutique, qui n'est pas destiné à la publication) les développements lyriques, élégiaques ou intimiste consacrés à l'Amour, à la Mort, aux épanchements du Moi. Valade et Verlaine dévoient la spiritualité de Baudelaire en racolage sordide:

La Mort des Amants

Nous aurons des lits pleins d'odeurs légères,

Des divans profonds comme des tombeaux

Et d'étranges fleurs sur des étagères,

Écloses pour nous sous des cieux plus beaux

Baudelaire

La Mort des cochons

Nous reniflerons dans les pissotières

Nous gougnotterons loin des lavabos

Et nous lécherons les eaux ménagères

Au risque d'avoir des procès-verbaux.

L. Valade, P. Verlaine ${ }^{18}$

Nouveau, Verlaine, Rimbaud, tournent en dérision les blasons du corps féminin auquel Albert Mérat se consacrait dans les sonnets de L'idole: à la componction du texte parodié, Nouveau substitue des allusions libertines, les deux autres compères une vulgarité sans retenue.Tous les trois font preuve d'une maîtrise prosodique qui dévoile sans pitié les afféteries de leur modèle:

Le sonnet des seins

L'éclosion superbe et jeune de ses seins

Pour enchaîner mes yeux fleurit sur sa poitrine.

Tels deux astres jumeaux dans la clarté marine

Palpitent dévolus aux suprêmes desseins.

A. Mérat

17 Le Parnassiculet contemporain, Recueil de vers nouveaux, précédé de L'Hôtel cu Dragon-bleu, Bassac, 1993 (reprint). Album zutique, fac-similé du manuscrit original, transcription et commentaires de P. Pia, Slatkine, 1981. Dixains réalistes, in G. Nouveau, La Doctrine de l'Amour, Poésie-Gallimard, édition de L. Forestier, 1981, p. 259-263. Les déliquescences. poèmes décadents d'Adoré Floupette, introduction et notes de N. Richard, A.-G. Nizet, 1984. 
Sonnet de la langue

Toute rose, à travers les dents blanches, que frange

L'épais rideau grenat de ses lèvres, écrin

De baisers sourds, en son caprice vipérin,

Sort, affinée, au bout, sa douce langue d'ange.

$$
\text { G. Nouveau }{ }^{19}
$$

Sonnet du Trou du Cul

Obscur et froncé comme un œillet violet

Il respire, humblement tapi parmi la mousse

Humide encore d'amour qui suit la fuite douce

Des fesses blanches jusqu'au coeur de son ourlet.

$$
\text { P. Verlaine, A. Rimbaud }{ }^{20}
$$

Les parodistes déployent une virtuosité qui démonte les procédures du langage poétique. Ils le désacralisent en donnant le spectacle de toutes les sortes d'acrobaties verbales et métriques: sonnet monosyllabique, rondeau "estrambote" où le dernier mot d'une strophe est repris au début de la suivante, Pan-Tho-Mètre (Tho est la divinité de la Terre, dans la mythologie égyptienne) de forme pyramidale qui termine, avant son "Épilogue", Le Parnassiculet contemporain $^{21}$, - et qu'E. Haraucourt, dix ans plus tard, dans $L a$ légende des sexes, inverse en un "Sonnet pointu" suggestif ("Reviens sur moi! Je sens ton amour qui se dresse", etc. ${ }^{22}$ ). Les pasticheurs sont, à en croire $\mathrm{Ch}$. Cros, "des soldats d'avant-garde" qui organisent une "Église des Totalistes" ${ }^{23}$. Ils conçoivent le travail du poète comme une élaboration formelle et rhétorique, rédigent des quatrains dont les vers ("holorimes") sont intégralement homophoniques deux à deux, ou des alexandrins palindromes dont les contraintes fascineront plus tard les ascètes de l'Oulipo:

\section{Léon, émir comu d'un roc, rime Noël}

Les jeunes poètes imitent François Coppée, auteur de dizains qui s'attachent à l'évocation des réalités familières: vie quotidienne, personnages issus du peuple, activités et objets ordinaires font entrer en poésie ce que les peintres appellent des "scènes de

19 Ibid., p. 185.

20 Ibid., p. 27.

21 Voir Le Parnassiculet contemporain, op. cit., p. 27, 23, 49.

22 E. Haraucourt, La Légende des sexes. Poèmes bystériques ou l'épopée des basventres [1883], Les Insolites, 1980, p. 47.

23 Charles Cros, CEuvres complètes, êdition établie par L. Forestier et P. Pia, J. J. Pauvert, 1964, p. 330-334. 
genre". Suivant le pas de la Muse que Coppée chausse de savates bourgeoises, ses plagiaires révèlent par l'outrecuidance de leur savoir-faire, qu'une prosodie sans éclat, loin de desservir la poésie, en renouvelle les consonances. Parce que vigilants, conscients de leurs procédures, surtout parce qu'écrits de mains de maîtres, leurs dizains élaborent une formule moqueuse qui se révèle régénératrice du genre. Car ils convertissent ce que François Coppée concevait comme une miniature émouvante en une représentation complexe dont la triple entente désigne à la fois un référent, un modèle littéraire et la distance qu'impose son énonciation. Il suffit de concentrer la platitude, de la "sur-écrire", si on peut dire, en accumulant les lieux communs et les "réclames" du jour, pour exprimer une saveur demeurée jusqu'alors inconnue:

II

Prisonnier d'un bureau, je connais le plaisir De goûter, tous les soirs, un moment de loisir. je rentre lentement chez moi, je me délasse Au cri des écoliers qui rentrent de la classe; Je traverse un jardin, où j'écoute, en marchant, Les adieux que les nids font au soleil couchant, Bruit pareil à celui d'une immense friture. Content comme un enfant qu'on promène en voiture, Je regarde, j'admire, et sens avec bonbeur Que j'ai toujours la foi naîve du flâneur.

F. Coppée, Promenades et intérieurs

II

J'ai du goût pour la flâne, et j'aime, par les rues, les réclames des murs fardés de couleurs crues, la Redingote Grise, et Monsieur Gallopau;

l'Hérissé qui rayonne au-dessous d'un chapeau; la femme aux cheveux faits de teintes différentes. Je m'amuse bien mieux que si j'avais des rentes avec l'homme des cinq violons à la fois, Bornibus, la Maison n'est pas au coin du Bois; le kiosque japonais et la colonne-affiche... Et je ne conçois pas le désir d'être riche.

Dixains réalistes ${ }^{24}$

24 Dixains réalistes, op. cit., p. 259-260. Dès Intimités (1868) qui comprend deux dizains sentimentaux, F. Coppée s'est fait le poète des humbles. Promenades et intérieurs (1871) compte trente-neuf dizains consacrés à des scènes de la vie quotidienne, d'une banalité sans faille, parfaitement "niaiseuse", 
La profanation du langage poétique le vide de sa substance conventionnelle, elle le soumet à des expériences qui le révèlent à lui-même. Elle recourt à toutes les sortes de références, de modèles et de tons: aux langages spécialisés, à la langue parlée, à la chanson - à l'excès, à l'excentricité, aux bizarreries.

Dès lors - écrit H. Scepi qui confronte la poésie de Mallarmé à celle des Fumistes de la fin du siècle - le discours n'est plus l'occasion d'un avènement décisif du sens, le lieu de l'expression intégrale et transparente d'un moi totalisant qui en serait l'ultime garantie, mais le procès d'un conflit à la fois jubilatoire et désenchanté, qui met aux prises les topoï du poétique avec l'implacable ironie du sujet qui toujours cherche à s'approprier la langue de l'Autre. ${ }^{25}$

Le retournement des valeurs ("l'absence de tout bouquet") s'opère sur le mode comique car il fait basculer l'initiation à l'absolu dans les chausse-trappes d'une parole vouée à l'écriture raffinée du rien, comme l'indiquent, par exemple, ces vers pompeux du Parnassiculet contemporain, "aussi remarquables par leur beauté que par leur incohérence":

Panthéisme

C'est le Milieu, la Fin et le Commencement,

Trois et pourtant Zéro, Néant et pourtant Nombre,

Obscur puisqu'il est clair et clair puisqu'il est sombre,

C'est Lui la Certitude et Lui l'Effarement. ${ }^{26}$

En discréditant la formulation de l'indicible, les parodistes conçoivent la poésie comme une activité déviante, une parole de transgression, une production verbale en quête d'elle-même. Ils la mettent à l'épreuve en posant un miroir déformant où le poète ne distingue de lui-même qu'une image brouillée et ne parvient plus à discerner l'objet de sa quête. Car la Poésie ne gît pas là où on pouvait le croire, là où les doctes la situent. Le rire n'inscrit pas l'ombre portée du "vrai » langage poétique, il en révèle les innombrables ressources.

comme on dit au Québec: "J'écris ces vers, ainsi qu'on fait des cigarettes", déclare l'auteur dans la dernière pièce de son recueil.

25 H. Scepi, "Une littérature excentrique: enjeux et ambiguités fin-de-siècle", Critique, no 587, avril 1996, p. 293.

26 Le Parnassiculet contemporain, op. cit., p. 6 et p. 31. 


\section{La subversion du rire par le poétique}

Sous des appellations variées, les bohèmes de la fin du siècle dernier ont remis la Poésie sur ses pieds, en l'incitant à emprunter des parcours inattendus. Dans une adresse à ses compagnons Hydropathes, Émile Goudeau la place à la croisée des chemins: "On médite des ouvrages philosophico-poétiques, en laissant audessus planer toutes ailes déployées, la fantaisie." 27 Son programme sera abondamment rempli dans les revues et dans les cabarets qui essaiment, à partir du Chat Noir, lieu de rencontres, ban d'essais et organe de presse. La poésie gaie multiplie les fables-express qui se présentent comme des histoires drôles mises en vers. Elle divulgue des "conseils utiles" pour faire disparaître les corps de ceux qu'on assassine ("L'Incinération ", Jules Jouy), elle commémore "Le Départ des asperges" dans des suites potagères de quatrains en octosyllabes (Hugues Delorme), elle cite les formules des manuels de Chimie ("Zéro, zéro, six, neuf, deux, six / Telle est de l'hydrogène / D'après Thénard et Regnault fils / La densité certaine...", K. Lomel), elle s'énonce sous la forme de monologues, de rengaines argotiques, de couplets interprétés devant un public complice qui ne demande qu'à reprendre en chœur les refrains:

Mourons ensemble

Pour être heureux;

La mort rassemble

Les amoureux!

Mac Nab, "Suicide en partie double", Poèmes incongrus ${ }^{28}$

Les productions que les poètes du Chat Noir, de Montmartre ou des gazettes parisiennes destinent au grand public, le réconcilient avec un mode d'expression qui se limite le plus souvent à des tirages confidentiels. Le courrier français, Le rire, La vie drôle, publient des poésies que leurs auteurs assemblent parfois en recueils. Mais cette audience est préservée au prix de concessions qui refrènent l'esprit de subversion. Les poètes au label "fantaisiste" sont des auteurs de fantaisie comme on le dit des produits

\section{L'Hydropathe, $\mathrm{n}^{\circ} 4,5$ mars 1879.}

28 Les poèmes de Jules Jouy ("L'Invention. Conseils utiles") et de Hugues Delorme ("Le Départ des asperges") ont été publiés par B. Millanvoye: Antbologie des poètes de Montmartre, Paris, 1909, p. 219 et p. 82 . Celui de K. Lomel dans Le chat noir du 6 mai 1882 (avec des suites dans les numéros du 16 octobre et du 25 novembre de la même année). 
d'imitation et des bijoux de deux sous car ils sont attachés à remplir une fonction sans inquiéter leur public. Ils produisent de la poésie "gaie" de manière aussi convenue qu’à la même époque les poètes du Mercure de France produisent des vers qu'imprègne l'idéal symboliste. Franc-Nohain, Maurice Donnay empruntent les tours d'un burlesque qui ne prend pas de risques. Et tel "Sonnet rustique" que publient, à quelques années de distance, Le chat noir puis Le rire, semble écrit par un François Coppée de la nouvelle génération, qui aurait tiré parti des moqueries dont il avait été la cible:

Quand la tomate, au soir, lasse d'avoir rougi,

Fuit le ruisseau jaseur que fréquente l'ablette,

J'aime écrire des mots commençant par des J

Sur l'ivoire bénin de mes humbles tablettes.

[...]

Alors, si le dîner n'est pas encore cuit,

Je décroche un fusil et je mange un biscuit

Avec mon perroquet sur le pas de ma porte...

$$
\text { George Auriol } 29
$$

La poésie peut ignorer les sujets "élevés " auxquels l'enseignement initiait les élèves (exposez les idées qu' éveille dans votre âme une promenade au bord de l'océan", indique le sujet d'une composition française dans un manuel des années $1880^{30}$ ). De fait, les poètes usent d'une gamme de tons où le sarcasme, le sourire, la blague, trouvent leur part obligée. Les fleurs du mal ne dédaignent pas le calembour et, dès l'adresse "Au lecteur", le recueil se constelle de majuscules ironiques. Tout en désignant les "Régions de la Poésie pure" (Fusées), Baudelaire discerne un "élément comique pur" dans la stylisation de la pantomime qu'il oppose au comique "significatif " de la comédie ${ }^{31}$. En s'attachant à l'utopie de la "pureté", les poètes se préservent des formulations galvaudées qui les dépossèdent d'un usage prestigieux du

29 Ce sonnet de G. Auriol a paru dans Le chat noir (9 mars 1884) puis dans Le Rire (26 octobre 1895). Je remercie F. Caradec de m'en avoir communiqué le texte et les références. On trouvera des échantillons de cette poésie de cabaret et de gazette dans D. Grojnowski et B. Sarrazin, L'esprit fumiste et les rires "fin de siècle, J. Corti, 1990; et dans A. Velter, Anthologie des poètes du Chat noir, Poésie-Gallimard, 1996.

30 A. Filon, Nouvelles narrations françaises, précédées d'exercices préparatoires, Paris, 1827. Ce manuel, destiné aux élèves de l'enseignement secondaire, connaît une $26^{\mathrm{e}}$ réédition en...1911!.

31 Charles Baudelaire, "De l'essence du rire ", CEurres, op. cit., p. 724. 
langage. Cette conception qu'on dira plus tard "intransitive", "autotélique", délimite un territoire de l'expression où les catégories génériques cessent de jouer un rôle dêcisif. Le principe d'autonomie affecte toutes les sortes d'écrits alors même que les traditions se perpétuent. Car les théâtres représentent toujours des pièces comiques, les revues littéraires des poèmes en vers ou en prose. Mais les ouvres sont hantées par une formule où les frontières se résorbent.

Depuis longtemps, du reste, les lecteurs ont fait l'expérience des intrications du poétique et du comique, dans des pages qui ne se présentent pas à eux sous des labels convenus: Les cbants de Maldoror, Contes cruels, "Mémoires d'un amnésique", pour ne citer que quelques titres exemplaires ${ }^{32}$. Une certaine exaltation du langage s'y démarque des jeux de mots et des traits d'esprit comme du primat de la musique et des images. Elle affirme la suprématie du Sujet, son aptitude à constituer de toutes pièces une réalité qui le pose en créateur d'absolu. Portés à leur point d'incandescence, le poétique et le comique y opèrent la fusion qui donne au sublime son expression moderne.

Les philosophies du Romantisme avaient de longue date revendiqué cette conquête par laquelle l'artiste "s'érige en maître de toute réalité, change à son gré l'ordre naturel des choses" et n'est satisfait que lorsque "les objets qui figurent dans son tableau, par [...] le caprice ou la verve humoristique, offrent un ensemble contradictoire, un spectacle fantastique où tout se heurte et se détruit "( Hegel $^{33}$ ). Attesté par des textes qui emprun-

32 Je signale ici, non sans arbitraire, mais pour le plaisir: Lautréamont, au quatrième des Chants de Maldoror, le deuxième paragraphe qui commence par: "Deux piliers, qu'il n'était pas difficile et encore moins possible de prendre pour des baobabs, s'apercevaient dans la vallée, plus grands que deux épingles "; dans le "conte cruel " de Villiers de l'Tsle-Adam intitulé "L'Inconnue *, la tirade finale de l'héroïne; et d'Erik Satie (outre "Ce que je suis", Mémoires d'un amnésique), ces observations qui figurent dans "Les raisonnements d'un têtu * au numéro 175 :

"Il y a des arbres sur lesquels vous ne verrez jamais un oiseau : les cèdres entre autres : ces arbres sont si sombres que les oiseaux s'ennuient sur eux et les évitent.

Les peupliers ne sont pas plus visités, car il est dangereux d'y accéder: ils sont beaucoup trop hauts."

(Écrits réunis par O. Volta, éditions G. Lebovici, 1990, p. 71 et p. 153).

33 Hegel, Cours d'esthétique, traduction française, en deux volumes de Ch. Bénard, Paris, 1840 et 1843 t. 2, p. 503 . Cet ensemble se termine par un cha- 
tent les configurations familières du poème, le comique affecte celui-ci d'un coefficient d'indistinction. La conjonction des effets produit un émoi qui n'appartient pas en propre à la poésie mais qui procède de tout usage sidérant du langage. Comme dans ce début de sonnet où Jarry exploite les réseaux prosodiques, phoniques, et métaphoriques pour que bruissent sur la page les feuilles hallucinées d'un arbre que nulle forêt ne recèle:

\section{VÉGÉTAL}

Le vélin écrit rit et grimace, livide.

Les signes sont dansants et fous. Les uns, flambeaux,

Pétillent radieux dans une page vide.

D'autres en rangs pressés, acrobates corbeaux,

Dans la neige épandue ouvrent leur bec avide.

Le livre est un grand arbre émergé des tombeaux.

Et ses feuilles, ainsi que d'un sac qui se vide,

Volent au vent vorace et parlent par lambeaux.

Les trois meubles du mage surannées, II

Les minutes de sable mémorial

Ou comme dans les dernières strophes de la série des "Contrerimes" où l'énigme de la vie et de la mort, les altercations du désir et de la rencontre, projettent leurs ombres portées sur trois strophes aux mètres brefs alternés. La miniature évoque comme par amusement le drame qui couve dans l'ardeur de l'instant. En éclairant d'un sourire quelques syllabes murmurées, le sublime de la fantaisie sollicite un mystère qui lui paraît sans aucun doute aussi insondable (et effrayant) que l'est la divinité pour l'âme romantique:

\section{LXX}

La vie est plus vaine une image

Que l'ombre sur le mur.

Pourtant l'hiéroglyphe obscur

Qu'y trace ton passage

M'enchante, et ton rire pareil

Au vif éclat des armes;

Et jusqu'à ces menteuses larmes

Qui miraient le soleil.

Mourir non plus n'est ombre vaine.

La nuit, quand tu as peur,

pitre consacré à la "Destruction de l'art romantique ". Pour Hegel le "Poète " a pour modèle Shakespeare, il recherche un adéveloppement personnel et subjectif " où "tout a une place". 
N'écoute pas battre ton cœur:

C'est une étrange peine.

Paul-Jean Toulet, Contrerimes

L'homme appréhende le mystère par le langage mais il revient au langage de lui révéler ses propres mystères car il lui communique autre chose que ce qu'il exprime: les effets qu'il gouverne toujours l'excèdent. Les mots du poème enregistrent l'énigme des significations divergentes, celle du sens infus et de l'inexprimable. Le végétal de Jarry, l'hiéroglyphe de Toulet ne ressortissent pas à l'expérience sensible et intelligible. Ils introduisent par effraction dans un espace insolite où l'ineffable a lieu. Dans la logique d'une esthétique négative, ce qui est dit n'est pas nécessairement concevable, ce qui est communiqué demeure virtuel. Les énoncés poéticomiques procèdent d'un sublime moderne qui outrepasse les bornes de la signification, ils obligent à concevoir l'inconcevable (est sublime, écrit Kant, un objet "dont la représentation détermine l'esprit à concevoir le fait que la nature est inaccessible en tant que présentation des idées" ${ }^{34}$ ). L'abbé Bremond attribuait le "caractère proprement poétique" du poème "à la présence, au rayonnement, à l'action transformante et unifiante d'une réalité mystérieuse "35. Aux adeptes de la poésie pure on dédiera les vocables que prononce Rrose Sélavy, la Muse des poètes ironistes qu'inspirent, eux aussi, les vertiges de l'ineffable:

Nous livrons à domicile: moustiques domestiques (demi-stock). Marcel Duchamp.

34 Kant, Critique de la faculté de juger ("Analyse du sublime"), Folio-essais, Gallimard, 1985, p. 211.

35 Ch. Bremond, op. cit., p. 16. 